\title{
Scattering of acoustic waves by movable lightweight elastic screens
}

\author{
A. Tadeu*, L. Godinho \\ Department of Civil Engineering, University of Coimbra, Polo II-Pinhal de Marrocos, Coimbra 3030-290, Portugal
}

Received 19 April 2002; revised 12 September 2002; accepted 11 November 2002

\begin{abstract}
This paper computes the insertion loss provided by movable lightweight elastic screens, placed over an elastic half-space, when subjected to spatially sinusoidal harmonic line pressure sources. A gap between the acoustic screen and the elastic floor is allowed. The problem is formulated in the frequency domain via the boundary element method (BEM). The Green's functions used in the BEM formulation permit the solution to be obtained without the discretization of the flat solid-ground interface. Thus, only the boundary of the elastic screen is modeled, which allows the BEM to be efficient even for high frequencies of excitation. The formulation of the problem takes into account the full interaction between the fluid (air) and the solid elastic interfaces.

The validation of the algorithm uses a BEM model, which incorporates the Green's functions for a full space, requiring the full discretization of the ground. The model developed is then used to simulate the wave propagation in the vicinity of lightweight elastic screens with different dimensions and geometries. Both frequency and insertion loss results are computed over a grid of receivers. These results are also compared with those obtained with a rigid barrier and an infinite elastic panel.

(C) 2003 Elsevier Science Ltd. All rights reserved.
\end{abstract}

Keywords: Elastic barrier; Sound propagation; Boundary element method

\section{Introduction}

Acoustic screens are widely used as a shield against the sound generated by road traffic, machinery and equipment. These acoustics screens are commonly made of lightweight material, particularly when they are designed to be movable. In these latter cases, the connection of the barrier to the floor is frequently loose and there may be an air gap in the vicinity of the ground surface.

The performance of the acoustic barriers is normally evaluated assuming them to be rigid panels, firmly bonded to the floor. However, this simplification leads to an overestimation of the performance of the acoustic screen, particularly at low frequencies. This paper evaluates how the elastic behavior of the material from which the barrier and the floor are made influences the insertion loss predicted for a movable acoustic screen. The thickness of a possible air gap between the barrier and floor is an important parameter in this work.

A number of simplified numerical approaches for modeling wave propagation in the vicinity of acoustic

\footnotetext{
* Corresponding author. Tel.: + 351-239-797-204; fax: + 351-239-797190.

E-mail address: tadeu@dec.uc.pt (A. Tadeu).
}

barriers have been proposed over the years. Several simplified models that define the diffraction effect on the edge of a barrier in a basic form are employed in engineering practice to compute the insertion loss provided by acoustic barriers [1-4].

Among the numerical techniques, the diffraction-based methods are frequently used to analyze sound propagation in the vicinity of acoustic barriers. Lam [5] proposed one such method to calculate the acoustic energy loss provided by simple, finite length, three-dimensional (3D) acoustic barriers. Muradali and Fyfe [6] subsequently extended this work and compared the results from $2 \mathrm{D}$ and $3 \mathrm{D}$ models. They also analyzed the effect of single and parallel barriers and simulated both coherent and incoherent line-sources.

More elaborate numerical algorithms, such as the boundary element method (BEM) or the finite element method (FEM), can be used to compute the acoustic scattering response of barriers more realistically. However, a major drawback of these techniques is that they require very costly computer resources, particularly for very high frequencies. A boundary integral equation technique was proposed by Filippi and Dumery [7] and Terai [8] to calculate the scattering of sound waves by thin rigid screens in an unbounded medium. Later, Kawai and Terai [9] 
extended this model to predict the sound attenuation by rigid barriers placed over a totally reflective floor.

Duhamel [10] used the BEM to compute the 3D sound pressure around a 2D acoustic barrier of constant but arbitrary cross-section located over a rigid ground. This technique employs spatial Fourier transformation along the direction for which the geometry of the barrier does not change, which allows the $3 \mathrm{D}$ response to be obtained as a summation of simpler 2D solutions. The Green's function used in the BEM model is defined using the image source technique. Duhamel and Sergent [11] incorporated absorption by the floor, and compared the numerical and experimental results. A 2D BEM model was used by Morgan, Hothersall and Chandler-Wilde [12] to assess the influence of the shape and absorbent surface properties on the performance of barriers against railway noise. Lacerda et al. [13] used a dual boundary element formulation for computing the $2 \mathrm{D}$ sound wave propagation in the vicinity of acoustic barriers placed over an infinite plane, in which both the ground and the barrier were absorptive. Later, Lacerda et al. [14] proposed the use of a dual boundary element formulation to compute the 3D sound propagation around an absorptive barrier. The Green's functions used allow the absorptive properties of the ground to be taken into account. Jean, Defrance and Gabillet [15] evaluated the performance of acoustic barriers placed in the presence of traffic noise, simulated using point pressure sources, and coherent and incoherent line sources. Responses for point sources are obtained by using Fourier-like transformations, which allow the 2D BEM models to be used. In this model, absorbing properties can be ascribed to both the acoustic barrier and the ground surface.

The BEM is probably the tool best suited for modeling wave propagation in unbounded media, because it automatically satisfies the far field radiation conditions and it only requires a description of the medium in terms of boundary elements at the material discontinuities.

The authors have already used the BEM to evaluate the influence of the $2 \mathrm{D}$ acoustic barriers on the sound pressure level in the vicinity of tall buildings when a 3D pressure source is excited (a 2.5D problem) [16]. In that work, the acoustic barriers and the buildings are assumed to behave like rigid barriers. The Green's functions used were defined by the method of images, automatically satisfying the boundary conditions for the building and the floor. Therefore, only the boundaries of the barriers needed to be discretized with boundary elements. The BEM was formulated in the frequency domain and time solutions were obtained by means of inverse Fourier transforms. The 3D solution was obtained as a summation of 2D solutions after performing a spatial Fourier transform in the direction in which the geometry does not vary $[10,16]$. This summation is obtained in a discrete form by considering an infinite number of virtual point sources equally spaced along the $z$-axis, and sufficiently distant from one another to avoid spatial contamination of the response [17]. The present paper extends the authors' work by modeling the ground and the barrier as elastic mediums, taking into account the full interaction between the fluid (air) and the solid. Furthermore, the BEM formulation used allows the connection between the barrier and the ground floor to be loose, so that an air gap between the base of the screen and the ground surface can be included. This model is used to simulate the propagation of sound waves in the vicinity of movable lightweight elastic screens when subjected to spatially sinusoidal harmonic line pressure sources.

The horizontal ground surface is not discretized since the Green's functions account for the required boundary conditions at this interface. Thus, only the barrier-limiting surfaces need to be discretized.

The BEM formulation employs the analytical Green's functions proposed previously by the authors for the steady state response of a homogeneous elastic half-space bounded by a fluid medium, when subjected to spatially sinusoidal harmonic line loads [18]. These solutions use the solid displacement potentials defined by the authors [19] to define the Green's functions for a harmonic (steady state) line load, with a sinusoidally varying amplitude in the third dimension, placed in an infinite medium. Those functions were found to be in full agreement with the solution for moving loads given earlier by Pedersen, Sánchez-Sesma and Campillo [20] and Papageorgiou and Pei [21]. The fluid potential is obtained via a technique similar to that used for the solid potentials. All potentials are written as a superposition of plane waves, according to the technique used first by Lamb [22] for the 2D case, and then by Bouchon [23] and Kim and Papageorgiou [24] when evaluating the three-space dimension field by means of a discrete wave number representation.

This paper first describes briefly the BEM formulation used to model lightweight elastic screens. This BEM algorithm incorporates 2.5D Green's functions for the steady state response of a homogeneous elastic half-space bounded by a fluid medium, when subjected to a spatially sinusoidal harmonic pressure line load placed in the fluid medium, which renders the discretization of the groundfluid interface unnecessary. The derivation of these $2.5 \mathrm{D}$ Green's functions is briefly described in Appendix B. A direct BEM, using Green's functions for an unbounded space, which requires the full discretization of all the boundaries, is used to validate responses. The proposed BEM model is then used to simulate the wave propagation in the vicinity of movable lightweight elastic screens. Different screen dimensions and air gap thicknesses between the barrier and the floor are modeled. The frequency and insertion loss responses are computed over a grid of receivers for different spatially sinusoidal harmonic line pressure sources. The results obtained for a rigid barrier and for an infinite panel are used as a reference for comparison. 


\section{BEM formulation}

A homogeneous fluid medium bounded by a $2 \mathrm{D}$ flat elastic homogeneous ground formation, contains a homogeneous elastic 2D inclusion, as shown in Fig. 1. The ground has density $\rho_{1}$, allowing a shear wave velocity of $\beta_{1}$ and a compressional wave velocity of $\alpha_{1}$, while the material of the inclusion allows a shear wave velocity of $\beta_{2}$ and a compressional wave velocity of $\alpha_{2}$, exhibiting density $\rho_{2}$. The fluid hosting the inclusion has density $\rho_{\mathrm{f}}$ and permits a compressional wave velocity $\alpha_{\mathrm{f}}$.

A point pressure source is placed in the fluid at position $\left(x_{0}, y_{0}, z_{0}\right)$, oscillating at frequency $\omega$. The incident pressure field can be expressed by

$$
\begin{aligned}
& \sigma^{\text {full }}(\omega, x, y, z) \\
& =\frac{A \exp \left(\mathrm{i} \frac{\omega}{\alpha_{\mathrm{f}}}\left(\alpha_{\mathrm{f}} t-\sqrt{\left(x-x_{0}\right)^{2}+\left(y-y_{0}\right)^{2}+\left(z-z_{0}\right)^{2}}\right)\right)}{\sqrt{\left(x-x_{0}\right)^{2}+\left(y-y_{0}\right)^{2}+\left(z-z_{0}\right)^{2}}}
\end{aligned}
$$

in which $A$ is the wave amplitude and $i=\sqrt{-1}$.

As the geometry of the problem does not change along one direction $(z)$, it can be solved as a summation of $2 \mathrm{D}$ problems, for varying effective wavenumbers [18]

$k_{\alpha_{\mathrm{f}}}=\sqrt{\frac{\omega^{2}}{\alpha_{\mathrm{f}}^{2}}-k_{z}^{2}}, \quad \operatorname{Im} k_{\alpha_{\mathrm{f}}}<0$

where $k_{z}$ is the spatial wavenumber along the $z$-direction, after Fourier transformation of the problem in this direction. The incident pressure field is then expressed by

$$
\begin{aligned}
& \hat{\sigma}^{\text {full }}\left(\omega, x, y, k_{z}\right) \\
& \quad=\frac{-\mathrm{i} A}{2} H_{0}^{(2)}\left(k_{\alpha_{\mathrm{f}}} \sqrt{\left(x-x_{0}\right)^{2}+\left(y-y_{0}\right)^{2}}\right) \mathrm{e}^{-\mathrm{i} k_{z} z}
\end{aligned}
$$

in which $H_{n}^{(2)}(\cdot)$ are second Hankel functions of order $n$. The incident field in this frequency wavenumber domain can be seen as a spatially sinusoidal harmonic pressure line load along the $z$-direction, placed in the fluid at $\left(x_{0}, y_{0}\right)$, oscillating at frequency $\omega$ (see Fig. 1).

By applying an inverse Fourier transform, the former 3D pressure field can be obtained as

$\sigma^{\text {full }}(\omega, x, y, z)=\int_{-\infty}^{\infty} \hat{\sigma}^{\text {full }}\left(\omega, x, y, k_{z}\right) \mathrm{e}^{-\mathrm{i} k_{z} z} \mathrm{~d} k_{z}$

This continuous integral can be discretized by assuming the existence of an infinite number of sources placed along the $z$-direction at equal intervals, $L$. Thus, the incident field can be written as

$\sigma^{\text {full }}(\omega, x, y, z)=\frac{2 \pi}{L} \sum_{m=-\infty}^{\infty} \hat{\sigma}^{\text {full }}\left(\omega, x, y, k_{z}\right) \mathrm{e}^{-\mathrm{i} k_{z} z}$

with $k_{z}=(2 \pi / L) m$. This equation converges and can be approximated by a finite sum of terms. The distance $L$ needs to be large enough to avoid spatial contamination. In addition, the analyses use complex frequencies that further reduce the influence of the neighboring fictitious sources.

Using this technique, the incident field generated by a point pressure load can be obtained as a discrete summation of 2D line loads with different values of $k_{z}$. In the same way, the scattered field originated by a point pressure load can be evaluated by solving a sequence of $2 \mathrm{D}$ problems. This problem is often referred to in the literature as a $2.5 \mathrm{D}$ problem, because the geometry is $2 \mathrm{D}$ and the source is $3 \mathrm{D}$.

A BEM formulation is used to calculate each of the 2D scattering fields generated by the inclusion and the ground floor. The formulation used here employs Green's functions, which take into account the presence of the horizontal ground solid-fluid interface. Therefore, only the boundaries of the inclusion need to be discretized by boundary elements. A detailed explanation of the BEM equations can be found in Refs. [25,26]. It is nevertheless important to state that the BEM solution requires the evaluation of the integral equations along the appropriately discretized

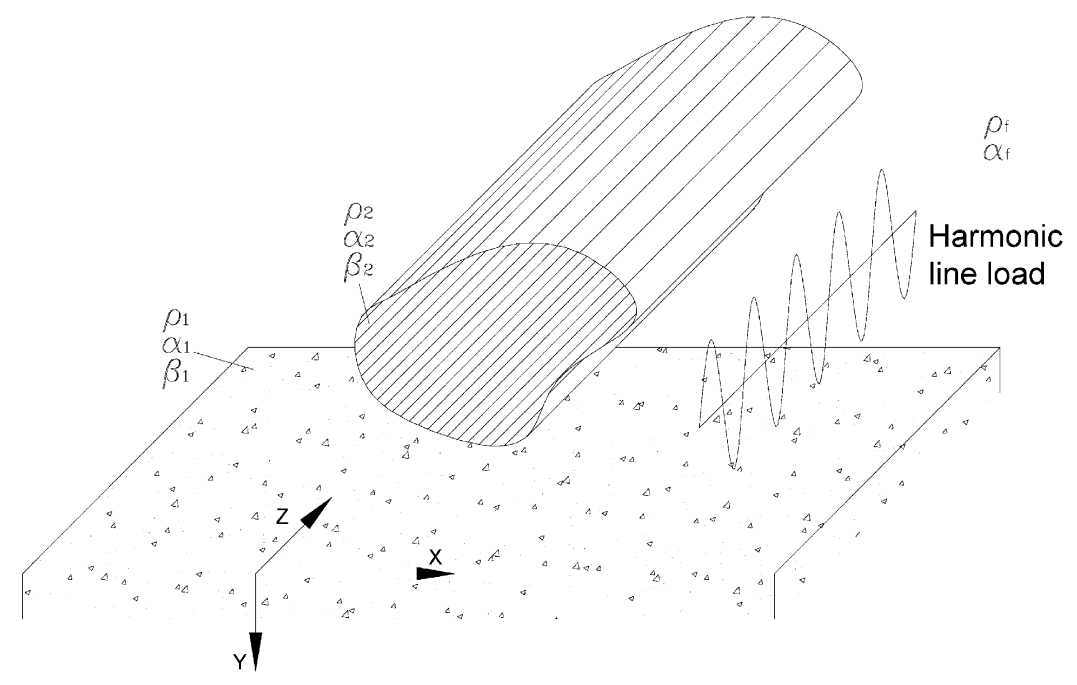

Fig. 1. Schematic representation of the BEM model. 
boundary of the elastic inclusion. If the boundary is discretized into $N$ straight boundary elements, with one nodal point in the middle of each element, and the existence of an incident pressure wavefield given by $\sigma^{\text {inc }}$ is assumed, the following integral equations can be defined:

along the solid domain

$$
\begin{aligned}
& \sum_{l=1}^{N} T_{i 1}^{(\mathrm{s}) k l} G_{i 1}^{(\mathrm{s}) k l}-\sum_{l=1}^{N}\left[u_{1}^{(\mathrm{s}) k l} H_{i 1}^{(\mathrm{s}) k l}+u_{2}^{(\mathrm{s}) k l} H_{i 2}^{(\mathrm{s}) k l}+u_{3}^{(\mathrm{s}) k l} H_{i 3}^{(\mathrm{s}) k l}\right] \\
& \quad=c_{k l} u_{i}^{(\mathrm{s}) k}
\end{aligned}
$$

$i=1,2,3$

along the fluid domain

$\sum_{l=1}^{N} T_{a_{1}}^{(\mathrm{f}) k l} G_{a_{1}}^{(\mathrm{f}) k l}-\sum_{l=1}^{N} u_{a_{1}}^{(\mathrm{f}) k l} H_{a_{1}}^{(\mathrm{f}) k l}+\sigma^{\mathrm{inc}}=c_{k l} u_{a_{1}}^{(\mathrm{f}) k}$

with $T_{i 1}^{(\mathrm{s}) k l}$ and $u_{j}^{(\mathrm{s}) k l}$ being the nodal tractions and displacements in the solid, $T_{a_{1}}^{(\mathrm{f}) k l}$ and $u_{a_{1}}^{(\mathrm{f}) k l}$ being the nodal tractions and displacements in the fluid

$H_{i j}^{(\mathrm{s}) k l}=\int_{C_{l}} H_{i j}^{(\mathrm{s})}\left(\omega, x_{k}, x_{l}, n_{l}\right) \mathrm{d} C_{l}, \quad i, j=1,2,3$

$H_{a_{1}}^{(\mathrm{f}) k l}=\int_{C_{l}} H_{a_{1}}^{(\mathrm{f})}\left(\omega, x_{k}, x_{l}, n_{l}\right) \mathrm{d} C_{l}$

$G_{i 1}^{(\mathrm{s}) k l}=\int_{C_{l}} G_{i 1}^{(\mathrm{s})}\left(\omega, x_{k}, x_{l}\right) \mathrm{d} C_{l}, \quad i=1,2,3$

$G_{a_{1}}^{(\mathrm{f}) k l}=\int_{C_{l}} G_{a_{1}}^{(\mathrm{f})}\left(\omega, x_{k}, x_{l}\right) \mathrm{d} C_{l}$

where $n_{l}$ is the unit outward normal for the $l$ th boundary segment $C_{l}$; the subscripts $i, j=1,2,3$ denote the normal, tangential and $z$ directions, respectively. In Eq. (6), $H_{i j}^{(\mathrm{s})}\left(\omega, x_{k}, x_{l}, n_{l}\right)$ and $G_{i j}^{(\mathrm{s})}\left(\omega, x_{k}, x_{l}\right)$ are, respectively, the Green's tensor for traction and displacement components in the elastic medium of the inclusion, at point $x_{l}$ in direction $j$, caused by a concentrated load acting at the source point $x_{k}$ in direction $i$. The factor $c_{k l}$ is a constant defined by the shape of the boundary, taking the value $1 / 2$ since the nodal point is in the middle of each straight element. The required twoand-a-half dimensional fundamental solution (Green's functions), and stress functions in Cartesian co-ordinates, are those for an unbounded elastic medium (listed in Appendix A).

$H_{a_{1}}^{(\mathrm{f})}\left(\omega, x_{k}, x_{l}, n_{l}\right)$ are the components of the Green's tensor for pressure in the fluid medium, at point $x_{l}$ caused by a pressure load acting at the source point $x_{k} ; G_{a_{1}}^{(\mathrm{f})}\left(\omega, x_{k}, x_{l}\right)$ are the components of the Green's tensor for displacement in the fluid medium, at point $x_{l}$ in the normal direction, caused by a pressure load acting at the source point $x_{k}$. As mentioned above, the formulation used here employs Green's functions that take into account the presence of the horizontal ground-fluid interface. These Green's functions are given by the sum of the incident field and the field generated at the solid-fluid interface, expressed by the so-called surface terms. The surface terms are defined so as to verify the required boundary conditions at this solidfluid interface, namely the continuity of normal displacements and stresses, and the null tangential stresses. Appendix B briefly explains how these fundamental solutions are arrived at. Note that imposing the continuity of normal particle velocities instead of normal displacements would lead to the same final results.

The integrations in Eq. (6) are evaluated using a Gaussian quadrature scheme, when they are not performed along the loaded element. For the loaded element, the existing singular integrands in the source terms of the Green's functions are calculated in closed form [27,28], while a Gaussian quadrature scheme is used to solve the integrands involving the surface terms originated at the ground-fluid interface.

The integral equations are manipulated and combined so as to impose the continuity of the normal displacements and normal stresses, and null shear stresses along the boundary of the inclusion, to establish a system of equations

$$
\left[\begin{array}{cccc}
G_{11}^{(\mathrm{s})} & -H_{11}^{(\mathrm{s})} & -H_{12}^{(\mathrm{s})} & -H_{13}^{(\mathrm{s})} \\
G_{21}^{(\mathrm{s})} & -H_{21}^{(\mathrm{s})} & -H_{22}^{(\mathrm{s})} & -H_{23}^{(\mathrm{s})} \\
G_{31}^{(\mathrm{s})} & -H_{31}^{(\mathrm{s})} & -H_{32}^{(\mathrm{s})} & -H_{33}^{(\mathrm{s})} \\
G_{a_{1}}^{(\mathrm{f})} & -H_{a_{1}}^{(\mathrm{f})} & 0 & 0
\end{array}\right]\left[\begin{array}{c}
T_{a_{1}}^{(\mathrm{f})} \\
u_{1}^{(\mathrm{s})} \\
u_{2}^{(\mathrm{s})} \\
u_{3}^{(\mathrm{s})}
\end{array}\right]=\left[\begin{array}{c}
0 \\
0 \\
0 \\
-\sigma^{\text {inc }}
\end{array}\right]
$$

In this system, $G_{i 1}^{(\mathrm{s})}, H_{i j}^{(\mathrm{s})}, G_{a_{1}}^{(\mathrm{f})}$ and $H_{a_{1}}^{(\mathrm{f})}$ are $N \times N$ submatrices generated by Eq. 6 , when the load is applied along the $N$ nodal points.

The solution of this system of equations gives the nodal displacements and tractions. Notice that the incident field in this system of equations is the sum of the source terms (twoand-a-half dimensional full-space) and the surface terms originated at the solid-fluid interface.

\section{BEM validation}

The results computed by the present BEM algorithm were compared with those given by a BEM model requiring the discretization of the ground-fluid interface, and the use of the Green's functions for a full space. The use of complex frequencies with a small imaginary part of the form $\omega_{\mathrm{c}}=$ $\omega-\mathrm{i} \eta$ (where $\eta=0.7(2 \pi / T)$ ) introduces a damping effect $[29,30]$, which allows the discretization of the ground-fluid interface in the BEM model to be limited.

Fig. 2 shows the geometry of the model used in the validation. A cylindrical circular elastic inclusion, with a radius of $1.0 \mathrm{~m}$, is inserted in a fluid medium bounded by an elastic ground medium with a horizontal flat surface. The mechanical properties of the elastic mediums forming the inclusion and ground, and those of the fluid medium are listed in Fig. 2.

A spatially sinusoidal harmonic pressure line load is applied at $x=0 \mathrm{~m}$ and $y=-0.5 \mathrm{~m}$. Computations are 


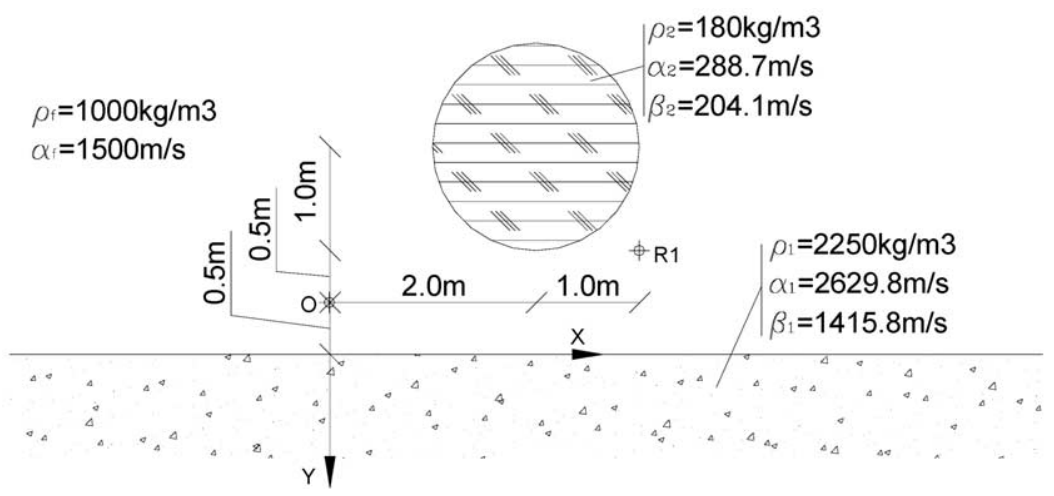

Fig. 2. Geometry of the problem.

achieved in the frequency domain $(2.50,320.0 \mathrm{~Hz})$ with a frequency increment of $2.5 \mathrm{~Hz}$. The imaginary part of the frequency has been set to $\eta=0.7(2 \pi / T)$, with $T=0.0466 \mathrm{~s}$.

Fig. 3 displays the real and imaginary parts of the scattered pressure field recorded by the receiver placed at $x=3.0 \mathrm{~m}$ and $y=-1.0 \mathrm{~m}$, for two different pressure line loads, $k_{z}=0$ and $1.5 \mathrm{rad} / \mathrm{m}$. Note that $k_{z}=0 \mathrm{rad} / \mathrm{m}$ corresponds to the pure 2D solution, while $k_{z}=1.5 \mathrm{rad} / \mathrm{m}$ corresponds to pressure waves that travel along the $z$ direction with an apparent velocity of $c=\omega / k_{z}$. The solid lines represent the proposed BEM model solutions, while the marked line corresponds to the BEM solution using the full
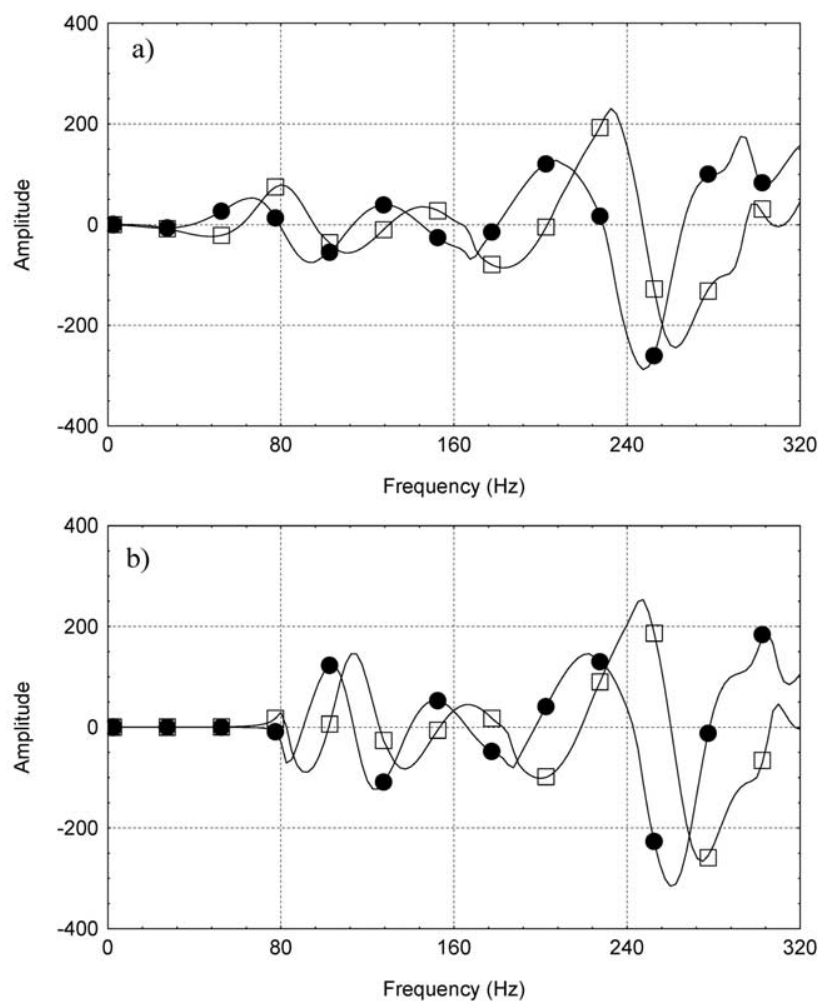

Fig. 3. Frequency domain response at receiver R1: (a) $k_{z}=0 \mathrm{rad} / \mathrm{m}$; (b) $k_{z}=1.5 \mathrm{rad} / \mathrm{m}$. boundary discretization of the ground-fluid interface. The square marks indicate the real part of the response, while the round marks refer to imaginary part.

The two solutions are in very close agreement for both loads, but the BEM solution using the full ground interface discretization required a very large number of boundary elements, and the use of significant damping. Equally good results were achieved from tests in which different loads and receivers were situated at different points.

\section{Movable lightweight elastic screens}

The BEM algorithm described above was used to compute the acoustic behavior of movable lightweight elastic screens when subjected to spatially sinusoidal harmonic line pressure sources. A gap between the acoustic screen and the ground floor is allowed. The solutions calculated for a rigid barrier are used as a reference to compare the results. This last problem is solved via BEM using the formulation described by the authors in an earlier work [16]. The response features are identified by comparing the results with those given by an elastic panel model bounded by two fluid media. These solutions were previously obtained analytically and can be found in Refs. [18,31].

Simulations are performed for sources with different spatial sinusoidal variation along the $z$-direction, that is, with different apparent wave velocities along the $z$-axis.

The screens' surfaces are modeled with a number of boundary elements that increases with the frequency excitation of the harmonic source. The ratio between the wavelength of the incident waves and the length of the boundary elements is kept to a minimum of six. The number of boundary elements used to model each screen is never less than 250. As the distance between the two vertical faces of the screen panel is small, the length of boundary elements modeling the screen is at least six times less than its thickness. To guarantee the accuracy of the results when there is an air gap, the length of the boundary elements modeling the base of the screen is at least four times less than the height of the air gap. 
In our examples, the ground is assumed to be made either of concrete $\left(\rho_{1}=2250 \mathrm{~kg} / \mathrm{m}^{3}, \beta_{1}=1415.8 \mathrm{~m} / \mathrm{s}\right.$ and $\left.\alpha_{1}=2629.8 \mathrm{~m} / \mathrm{s} \quad[32]\right)$ or cork $\left(\rho_{1}=180 \mathrm{~kg} / \mathrm{m}^{3}\right.$, $\beta_{1}=204.1 \mathrm{~m} / \mathrm{s}$ and $\alpha_{1}=288.7 \mathrm{~m} / \mathrm{s}$ [32]), while the screen is always modeled with cork $\left(\rho_{2}=180 \mathrm{~kg} / \mathrm{m}^{3}\right.$, $\beta_{2}=204.1 \mathrm{~m} / \mathrm{s}$ and $\left.\alpha_{2}=288.7 \mathrm{~m} / \mathrm{s}\right)$. The host acoustic medium had a pressure wave velocity of $\alpha_{\mathrm{f}}=340 \mathrm{~m} / \mathrm{s}$ and a density of $\rho_{\mathrm{f}}=1.22 \mathrm{~kg} / \mathrm{m}^{3}$ [32]. An acoustic harmonic line source is placed $0.6 \mathrm{~m}$ above the ground and $2.785 \mathrm{~m}$ from an acoustic screen of height $h$ with an air gap of thickness $w$. Computations are performed in the frequency domain (2.0, $2000.0 \mathrm{~Hz}$ ). The imaginary part of the frequency has been set to $\eta=3.5 \pi \mathrm{rad} / \mathrm{s}$.

The remainder of this section is divided into two parts. In the first part, the insertion loss provided by the elastic acoustic screen is computed. The response is computed along a grid of 25 receivers placed on the side of the acoustic screen not containing the source (see Fig. 4). The computations are performed both with and without the screen, to assess the reduction in sound pressure level that it provides. First, the acoustic source is $2 \mathrm{D}\left(k_{z}=0.0 \mathrm{rad} / \mathrm{m}\right)$, generating an incident field that travels perpendicular to the $z$-direction. Next, the acoustic source is assumed to generate waves with $k_{z} \neq 0.0 \mathrm{rad} / \mathrm{m}$, which can be seen as waves traveling along the $z$-direction with a certain inclination, defining an apparent wave velocity $c=\omega / k_{z}$. All these results are compared with those obtained with a rigid barrier and with an infinite elastic panel. In the second part, individual frequency results are computed over a finer grid of receivers, $0.2 \mathrm{~m}$ apart both horizontally and vertically, on either side of the acoustic screen, to analyze the sound pressure level registered in the presence of the acoustic barrier, at specific frequencies. The results obtained with a rigid acoustic screen are used as a reference.

\subsection{Insertion loss results}

The model for the first set of simulations describes an acoustic screen $t=0.15 \mathrm{~m}$ thick, and with $h+w=3.0 \mathrm{~m}$, when subjected to sound waves generated by a spatially sinusoidal harmonic line pressure source, with $k_{z}=0.0$ $\mathrm{rad} / \mathrm{m}$ (see Fig. 4). Fig. 5(a) displays the average insertion loss provided by the elastic and the rigid screens when there is an air gap $w=0.05 \mathrm{~m}$. The average insertion loss is obtained by taking the difference between the average sound pressure levels computed over the grid of receivers defined in Fig. 4, with and without an acoustic screen. In the low frequency range, the two curves are similar, but it can be seen that the rigid screen model outperforms the elastic screen. The results for the elastic screen show a set of dips in the insertion loss curve, which are not visible when the screen is modeled as a rigid body. They are caused by the interaction of different waves within the elastic material of the screen, and some of them are very marked, indicating that the elastic screen performs poorly at these frequencies. In order to better understand this behavior, an infinite panel made of cork, and with the same thickness, was modeled.

A grid of receivers is placed on the side of the panel not containing the source, as in Fig. 6(a). The average insertion loss is again computed as the average sound pressure level difference, obtained over the grid of receivers defined in the same figure, with and without the presence of the infinite wall.

The average insertion loss obtained for this model is represented in Fig. 6(b). It can be seen that the average insertion loss provided by the wall exhibits pronounced dips of insulation related to the multiple interactions of waves within the wall panel, such as those associated with the resonance effect. The first and second resonance frequencies occur at $f_{\mathrm{r} 1}=\alpha_{2} /(2 t)=960 \mathrm{~Hz}$ and $f_{\mathrm{r} 2}=2 \alpha_{2} /(2 t)=1920$ $\mathrm{Hz}$. The results found for the elastic screen indicate the appearance of insertion loss dips at frequencies similar to those associated with the resonance phenomena described above. This suggests that this behavior has a considerable effect on the insertion loss provided by an elastic screen. The insulation dips are very high and are not predicted by the rigid model.

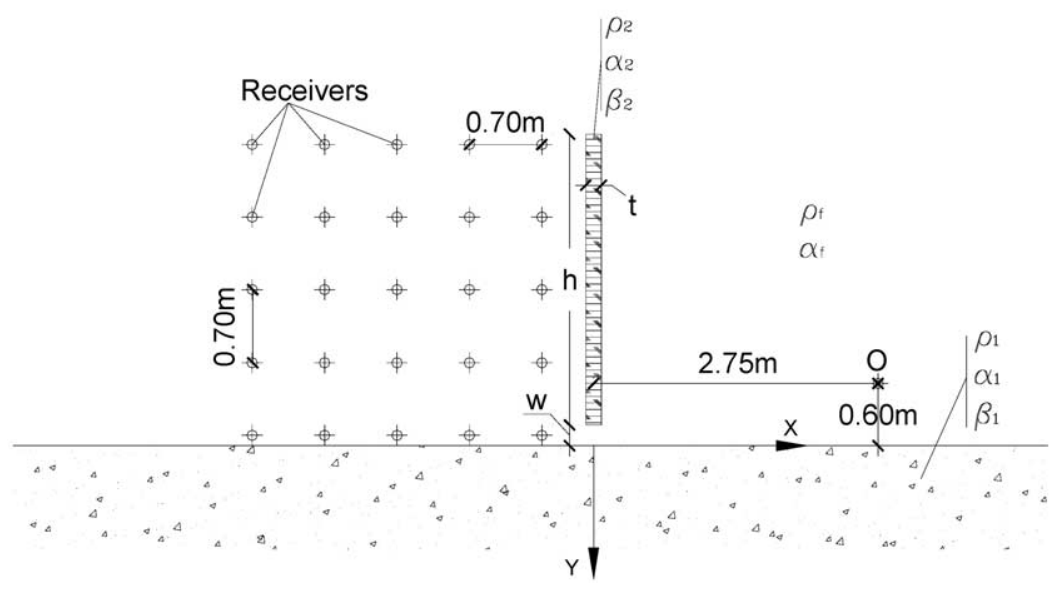

Fig. 4. Simulated model and grid of receivers used for insertion loss calculations. 

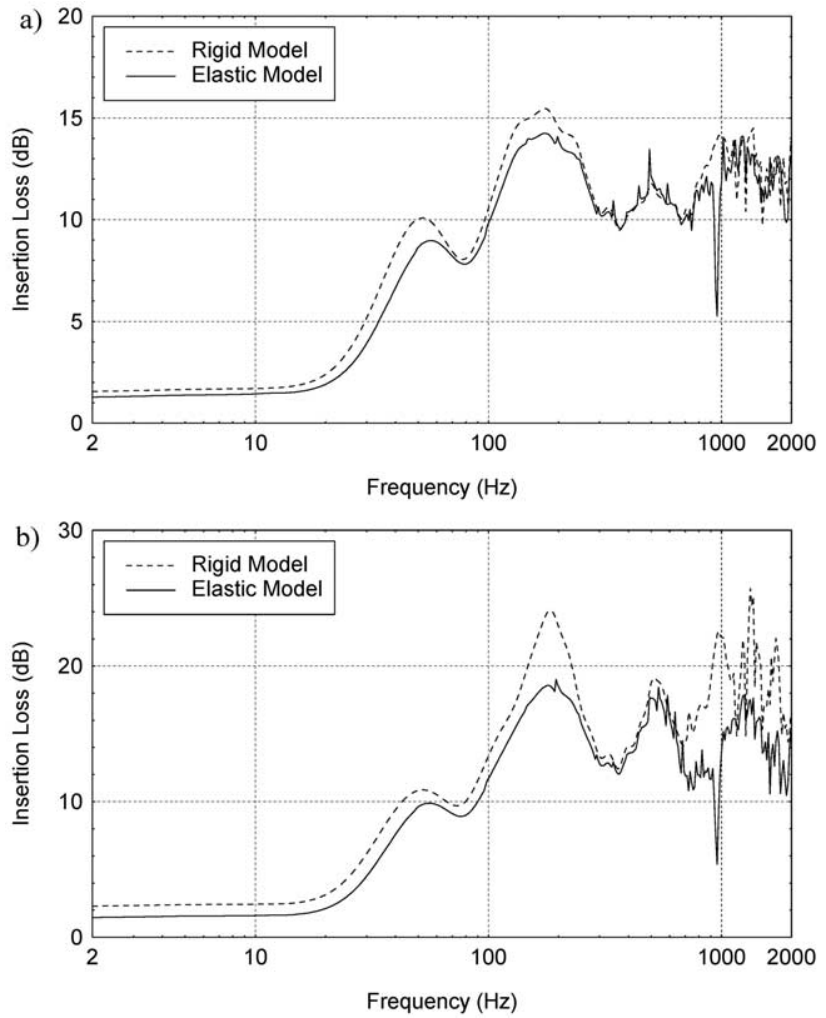

Fig. 5. Average insertion loss for $h+w=3.0 \mathrm{~m}$ : (a) elastic versus rigid screens with $w=0.05 \mathrm{~m}$; (b) elastic versus rigid screens with $w=0.02 \mathrm{~m}$.

Fig. 5(b) presents the results obtained when $w=0.02 \mathrm{~m}$. The rigid screen model again predicts higher insertion losses for the full domain of frequencies analyzed. Comparing these results with those when $w=0.05 \mathrm{~m}$, it can be seen that the smaller air gap allows a significant increase in the average insertion loss curve over the full frequency range. It can thus be concluded that the size of the air gap near the floor is a determinant factor in the final insertion loss provided by the screen.

The average insertion loss curves computed for taller screens, where $h+w=4.0 \mathrm{~m}$, and the $2 \mathrm{D}$ source is the same, are shown in Fig. 7. Fig. 7(a) and (b) refers to screens with air gaps of $w=0.05$ and $0.02 \mathrm{~m}$, respectively. As expected, the performance of both the rigid and the elastic screens is now better than that provided by the smaller screens, defined by $h+w=3.0 \mathrm{~m}$. This behavior is particularly important at lower frequencies. However, as the frequency increases, the gain in performance decreases, and the average insertion loss curve approaches that shown in Fig. 5(a) and (b). When the air gap is $w=0.02 \mathrm{~m}$ (Fig. 7(b)), there is an overall increase in the insertion loss provided by the screen. Again, this behavior seems to indicate that the size of the air gap has a marked influence on the average insertion loss provided by the screen.

Another simulation, also assuming a pure 2D scenario, was performed to study how the ground material influences the insertion loss provided by the elastic screen. Fig. 8(a) gives the results computed when the elastic ground has
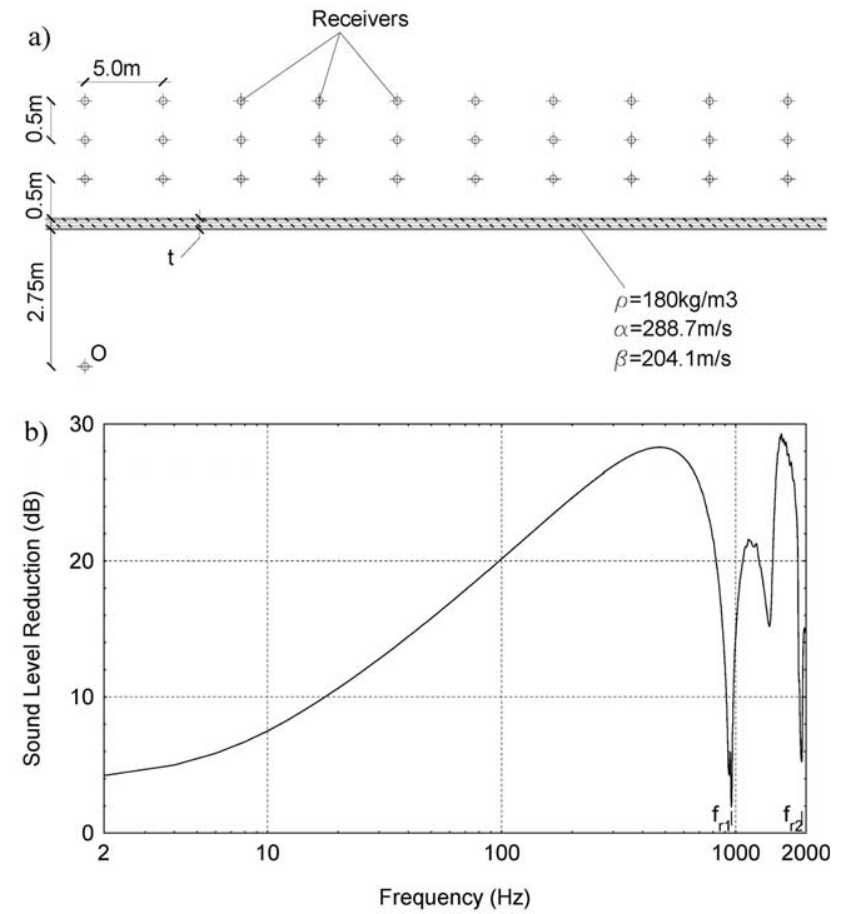

Fig. 6. Infinite elastic panel: (a) geometry of the model; (b) insertion loss curve.

the same properties as the elastic screen. This figure also includes the insertion loss curve computed when the ground is made of concrete. The insertion loss computed at low frequencies is similar for the two cases. Meanwhile, at high
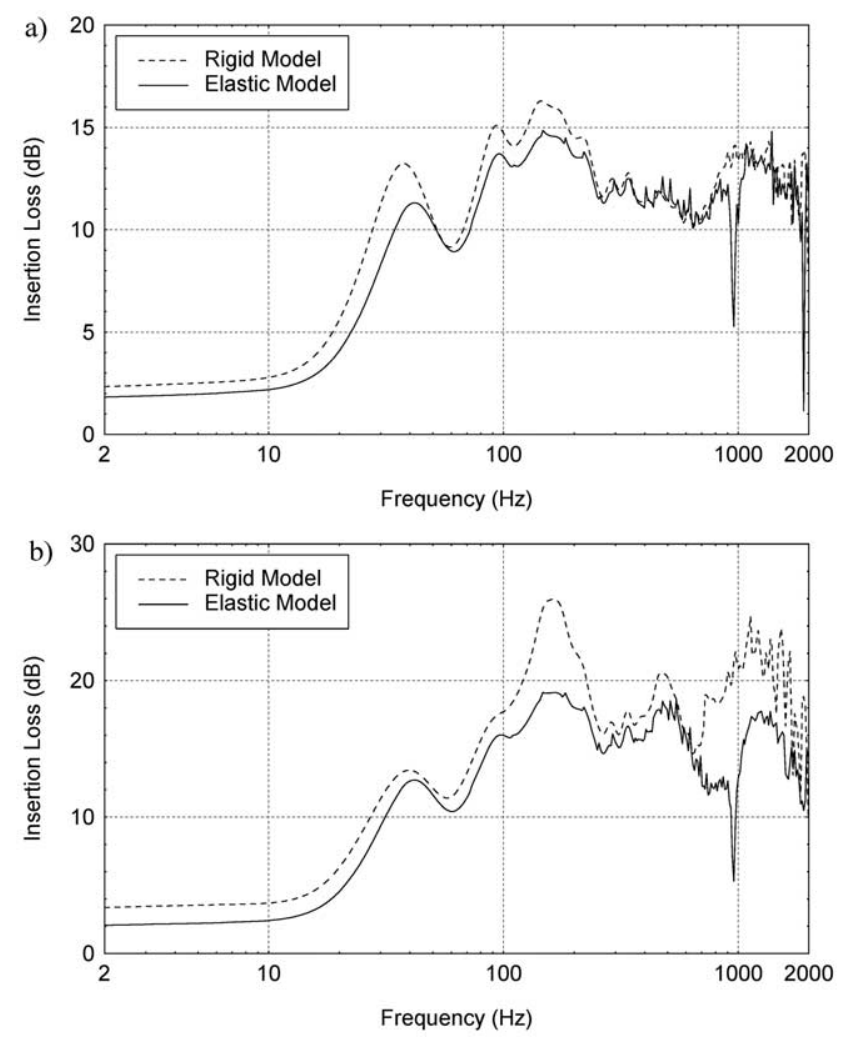

Fig. 7. Average insertion loss for $h+w=4.0 \mathrm{~m}$ : (a) elastic versus rigid screens with $w=0.05 \mathrm{~m}$; (b) elastic versus rigid screens with $w=0.02 \mathrm{~m}$. 
frequencies, the insertion loss differences become apparent. Since the scale of the plots does not allow these differences to be identified, an enlargement of the high frequency part of the response is shown in Fig. 8(b). However, the final insertion loss does not seem to be much influenced by the type of material of the ground, given the small differences registered.

The last simulation in this section corresponds to the case of an acoustic source emitting waves with an apparent velocity of $450 \mathrm{~m} / \mathrm{s}$ along the $z$-direction. This apparent velocity corresponds to waves with an inclination of $40.93^{\circ}$ in relation to the $z$-axis. Fig. 9(a) gives the results obtained when $h+w=3.0 \mathrm{~m}$ and $w=0.05 \mathrm{~m}$. Once more, these results are compared with those found for an infinite panel with the same thickness as the elastic screen (Fig. 9(b)).

The average insertion loss found for this situation is slightly lower than that registered for the 2D case (Fig. 5(a)). However, the main features identified for the case of infinite apparent velocity are maintained, and the average insertion loss curve exhibits marked insulation dips in frequencies where the resonance effect occurs within the elastic material.

\subsection{Individual frequency results}

A second set of results gives the sound pressure level evaluated over a fine grid of receivers, placed on either side of the acoustic screen, for specific frequencies. The sound pressure level is calculated by the expression
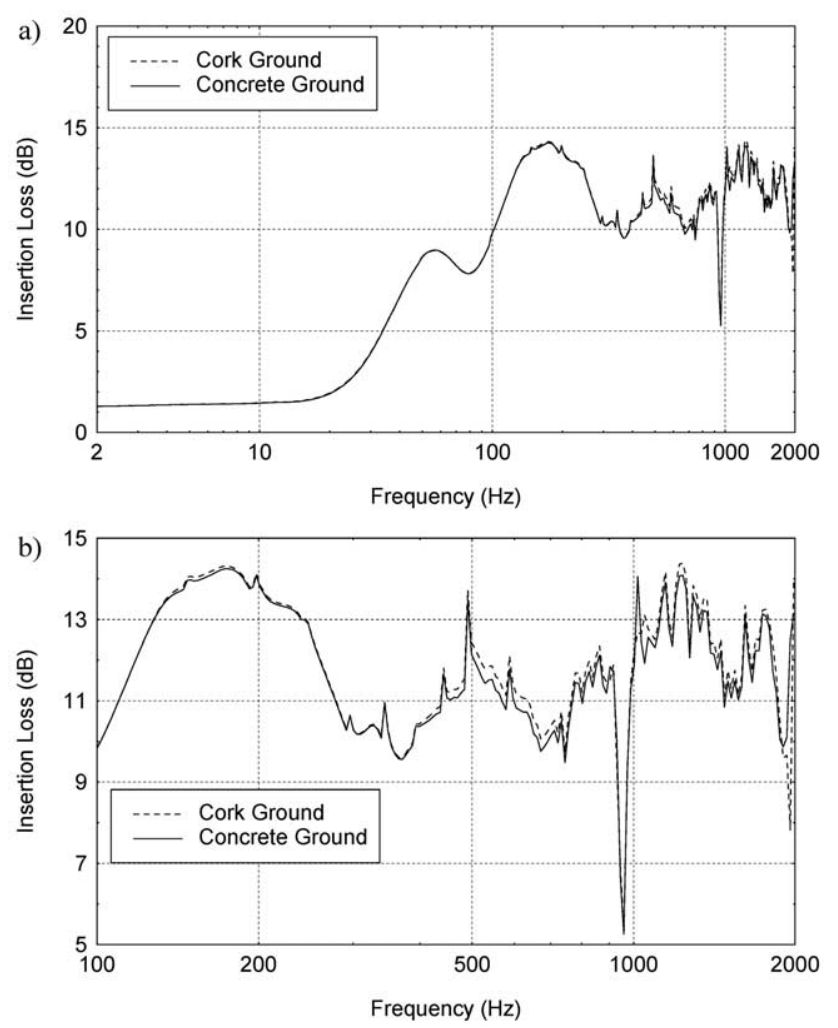

Fig. 8. Average insertion loss computed when the ground is made from different elastic materials (cork and concrete): (a) frequency range (2.0, $2000.0 \mathrm{~Hz})$; (b) frequency range $(100.0,2000.0 \mathrm{~Hz})$.
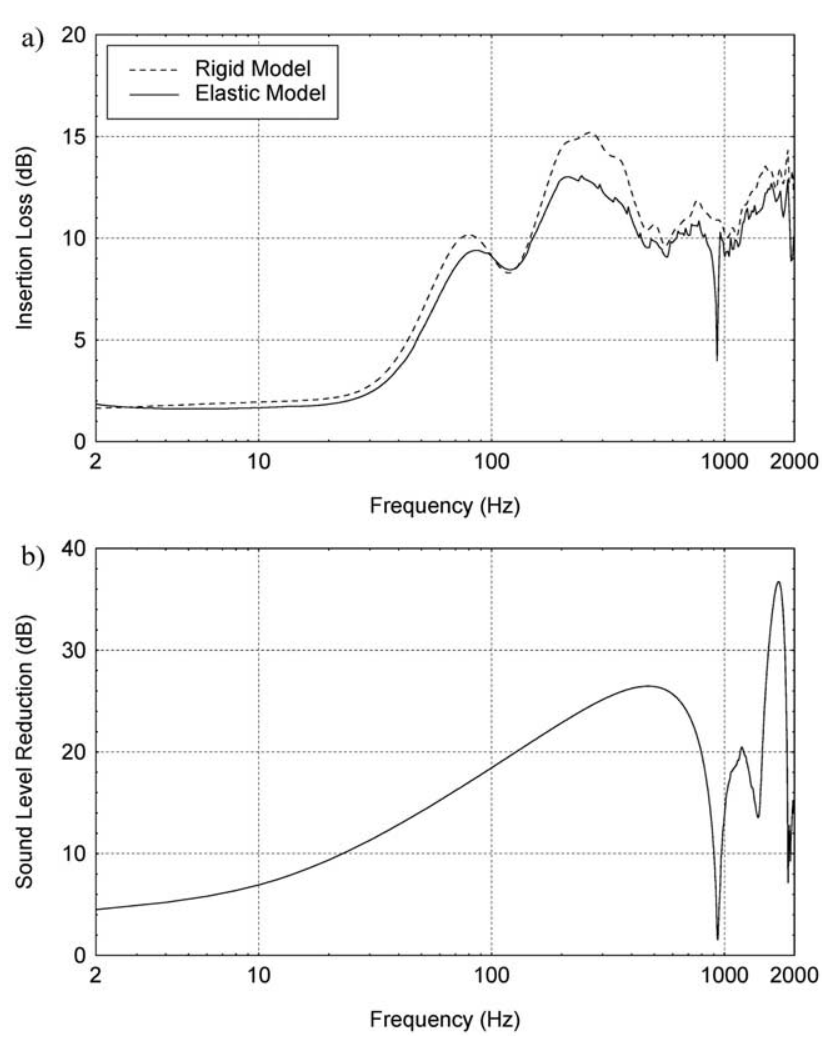

Fig. 9. Average insertion loss when the apparent velocity is $450 \mathrm{~m} / \mathrm{s}$ : (a) elastic versus rigid screens with $w=0.05 \mathrm{~m}$; (b) infinite elastic panel.

$10 \log \left[p^{2} /\left(2 \times 10^{-5}\right)^{2}\right\rfloor$, where $p$ refers to the pressure amplitude and $2 \times 10^{-5}$ is a pressure of reference.

Fig. 10 displays the sound pressure level at each receiver on a dB scale, for $50.0,500.0,960.0 \mathrm{~Hz}$, in the presence of either an elastic (Fig. 10(a)) or a rigid screen (Fig. 10(b)). These plots use a gray scale, ranging from black to white as the amplitude increases. At a frequency of $50.0 \mathrm{~Hz}$ the two results are similar, except at the receivers placed behind the screen, near to the ground and in the close vicinity of the screen. When elastic ground is simulated, the sound pressure level behind the screen in the vicinity of the ground is attenuated, since this does not permit the perfect reflection of the sound that the rigid floor does. As the frequency increases, the behavior of the response provided by the two models becomes similar $(500.0 \mathrm{~Hz})$. The results show that the presence of a small air gap at the base of the screen further increases the global sound pressure level registered behind it. However, when the chosen frequency coincides with the first dip related to the resonance effect inside the elastic material of the screen $(960.0 \mathrm{~Hz})$, the elastic screen model exhibits high sound pressure levels at receivers placed behind the screen. This is because the resonance effect arising within the elastic panel allows sound energy to travel through it with only small losses. By contrast, the insertion loss computed for the rigid model reveals a pronounced attenuation behind the acoustic screen. This result was anticipated, since all the energy hitting its surface is reflected back. Thus, the only energy reaching the receivers placed behind the barrier is that 
a)
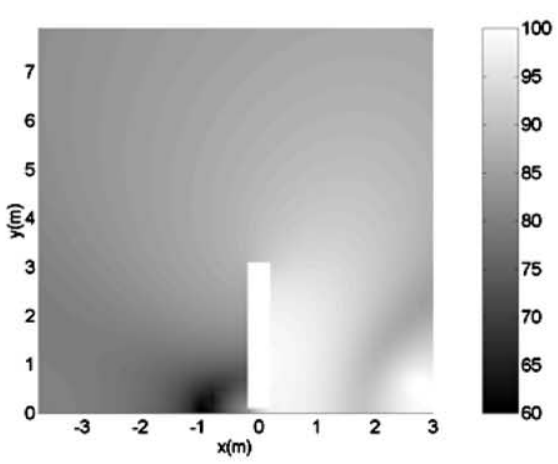

b)

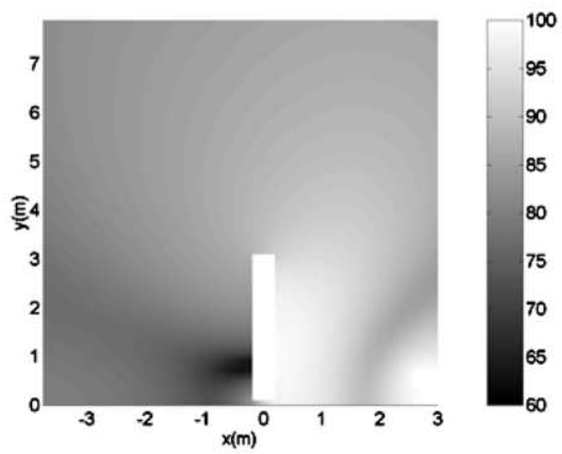

$500.0 \mathrm{~Hz}$
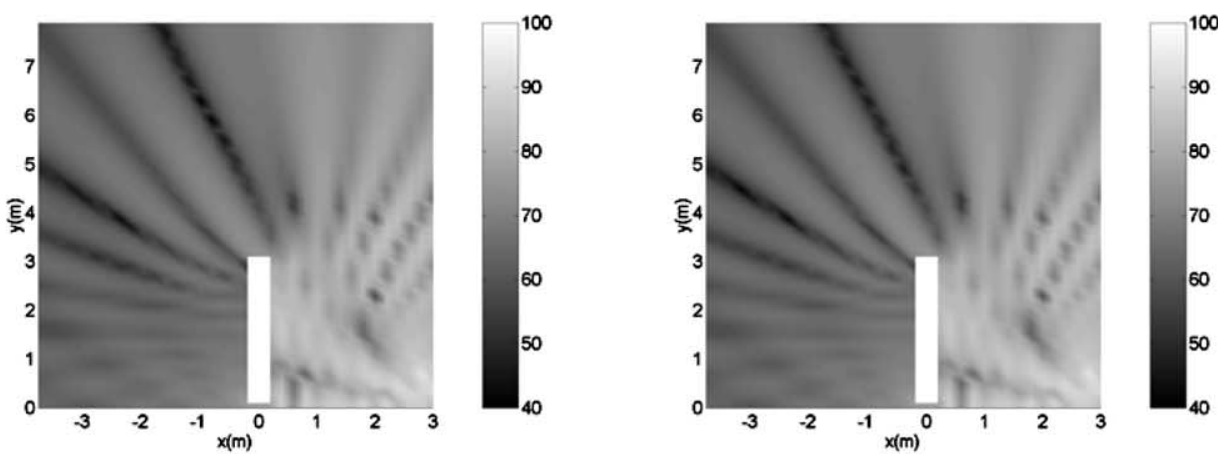

$960.0 \mathrm{~Hz}$
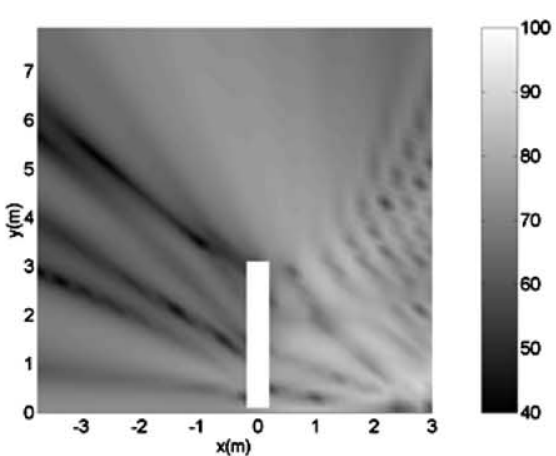

$50.0 \mathrm{~Hz}$

90

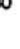

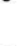

50

.

Fig. 10. Sound pressure level computed over a grid of receivers for individual frequencies, when $h+w=3.0 \mathrm{~m}$ and $w=0.05 \mathrm{~m}$ : (a) elastic screen model; (b) rigid screen model.

which is diffracted by its top edge, and that passing through the air gap at its base. In fact, a distinct increase in the sound pressure level near the base of the rigid screen can be seen in Fig. 10(b), and this is due to the presence of the air gap.

\section{Conclusions}

This paper studied the behavior of movable elastic screens via BEM. The proposed BEM model was found to be efficient, because only the surface of the inclusion needs to be discretized, since the Green's functions used take the full fluid-solid interaction at the ground surface into account.

The computed results were compared with those provided by a rigid model, solved using the BEM. The insertion loss provided by the elastic screen appeared to be highly dependent on the dynamic behavior of the screen, exhibiting pronounced dips related to the resonance effects originated by the interaction of waves within the panel. The responses for individual frequencies enabled this behavior to be clearly identified. Outside these zones the insertion 
loss provided by the elastic screen appears to be similar to that of the rigid barrier.

The size of the gap was found to have an important effect on the performance of the screen. Even a small gap contributes to the enhancement of the sound pressure level behind the screen.

The simulations presented assumed the existence of a lightweight cork screen. Similar dynamic behavior is anticipated when the elastic material of the screen or its thickness is changed. Thus, the behavior will be similar to the rigid model except in the vicinity of the eigenmodes of the dynamic system, such as those associated with resonance frequencies.

\section{Appendix A. The 2.5D Green's functions for an unbounded formation}

\section{A.1. Elastic formation}

Definitions:

$\lambda_{2}, \mu_{2} \quad$ Lamé constants

$\rho_{2} \quad$ Mass density

$\alpha_{2}=\sqrt{\left(\lambda_{2}+2 \mu_{2}\right) / \rho_{2}}$ P wave velocity

$\beta_{2}=\sqrt{\mu_{2} / \rho_{2}} \mathrm{~S}$ wave velocity

$k_{\mathrm{p} 2}=\omega / \alpha_{2}$

$k_{\mathrm{s} 2}=\omega / \beta_{2}$

$k_{\alpha 2}=\sqrt{k_{\mathrm{p} 2}^{2}-k_{z}^{2}}$

$k_{\beta 2}=\sqrt{k_{\mathrm{s} 2}^{2}-k_{z}^{2}}$

$r=\sqrt{\left(x-x_{0}\right)^{2}+\left(y-y_{0}\right)^{2}}$

$A=1 / 4 \mathrm{i} \rho_{2} \omega^{2}$ Amplitude

$\gamma_{i}=\partial r / \partial x_{i}=x_{i} / r \quad i=1,2$ Direction cosines

$H_{n \alpha}=H_{n}^{(2)}\left(k_{\alpha 2} r\right) \quad H_{n \beta}=H_{n}^{(2)}\left(k_{\beta 2} r\right)$ Hankel functions

$B_{n}=k_{\beta 2}^{n} H_{n \beta}-k_{\alpha 2}^{n} H_{n \alpha} \quad B_{n}$ functions

Green's functions for displacements:

$$
\begin{aligned}
G_{x x} & =A\left[k_{\mathrm{s} 2}^{2} H_{0 \beta}-\frac{1}{r} B_{1}+\gamma_{x}^{2} B_{2}\right] \\
G_{y y} & =A\left[k_{\mathrm{s} 2}^{2} H_{0 \beta}-\frac{1}{r} B_{1}+\gamma_{y}^{2} B_{2}\right] \\
G_{z z} & =A\left[k_{\mathrm{s} 2}^{2} H_{0 \beta}-k_{z}^{2} B_{0}\right] \\
G_{x y} & =G_{y x}=\gamma_{x} \gamma_{y} A B_{2} \\
G_{x z} & =G_{z x}=\mathrm{i} k_{z} \gamma_{x} A B_{1} \\
G_{y z} & =G_{z y}=\mathrm{i} k_{z} \gamma_{y} A B_{1}
\end{aligned}
$$

The mathematical derivation of these Green's functions, expressions for the strains and stresses are given in Ref. [19].

\section{A.2. Fluid formation}

Definitions:

$\lambda_{\mathrm{f}} \quad$ Lamé constant

$\rho_{\mathrm{f}} \quad$ Mass density

$\alpha_{\mathrm{f}} \quad \mathrm{P}$ wave velocity

$k_{\mathrm{p}_{\mathrm{f}}}=\omega / \alpha_{\mathrm{f}}$

$k_{\alpha_{\mathrm{f}}}=\sqrt{k_{\mathrm{p}_{\mathrm{f}}}^{2}-k_{z}^{2}} \quad$ with $\operatorname{Im} k_{\alpha_{\mathrm{f}}} \leq 0$

$A_{\mathrm{f}}=-\mathrm{i} / 4$ Amplitude

$r=\sqrt{\left(x-x_{0}\right)^{2}+\left(y-y_{0}\right)^{2}}$

$\gamma_{i}=\partial r / \partial x_{i}=x_{i} / r \quad i=1,2$ Direction cosines

$H_{n \alpha_{\mathrm{f}}}=H_{n}^{(2)}\left(k_{\alpha_{\mathrm{f}}} r\right)$ Hankel functions

Green's functions for displacements:

$$
\begin{aligned}
& G_{\mathrm{f} x}=-A_{\mathrm{f}} k_{\alpha_{\mathrm{f}}}\left(-\frac{\alpha_{\mathrm{f}}^{2}}{\omega^{2} \lambda_{\mathrm{f}}}\right) H_{1 \alpha_{\mathrm{f}}} \gamma_{x} \\
& G_{\mathrm{f} x}=-A_{\mathrm{f}} k_{\alpha_{\mathrm{f}}}\left(-\frac{\alpha_{\mathrm{f}}^{2}}{\omega^{2} \lambda_{\mathrm{f}}}\right) H_{1 \alpha_{\mathrm{f}}} \gamma_{y}
\end{aligned}
$$

\section{Appendix B. The 2.5D Green's functions for a flat solid-fluid interface}

Consider a homogeneous fluid medium of infinite extent, bounded by a flat homogeneous elastic medium. This system is subjected at point $\left(x_{0}, y_{0}\right)$ to a spatially sinusoidal harmonic pressure line source along the $z$-direction.

The solution for this load can be calculated by adding the incident field generated by a unit pressure load to the field generated at the solid-fluid interface, expressed by the socalled surface terms. These surface terms are obtained as a function of solid displacement and fluid pressure potentials. The amplitudes of these potentials are defined so as to verify the required boundary conditions at this solid-fluid interface, namely the continuity of normal displacements and stresses, and null tangential stresses. Throughout this procedure the incident field and the surface terms must be expressed as an integral of plane waves. In order to transform this integral into a summation, it is assumed that there is an infinite number of sources distributed along the $x$ direction, at equal intervals $L_{x}$.

A full description of the mathematical derivation of this solution can be found in Ref. [18]. Only the potentials associated with the source, the surface terms and the final Green's function are given here.

The source term can be obtained making use of the dilatational potential

$$
\begin{aligned}
& \phi_{\text {fluid }}\left(\omega, x, y, k_{z}\right) \\
& =\frac{-\mathrm{i}}{4}\left(-\frac{\alpha_{\mathrm{f}}^{2}}{\omega^{2} \lambda_{\mathrm{f}}}\right) H_{0}^{(2)}\left(k_{\alpha_{\mathrm{f}}} \sqrt{\left(x-x_{0}\right)^{2}+\left(y-y_{0}\right)^{2}}\right)
\end{aligned}
$$


which can be written in the form

$\phi_{\text {fluid }}=-\frac{\mathrm{i}}{2 L_{x}} \sum_{n=-N}^{n=+N}\left[\left(\frac{-\alpha_{\mathrm{f}}^{2}}{\omega^{2} \lambda_{\mathrm{f}}}\right) \frac{E_{\mathrm{f}}}{v_{n}^{\mathrm{f}}}\right] E_{\mathrm{d}}$

where $E_{\mathrm{f}}=\mathrm{e}^{-\mathrm{i} v_{n}^{\mathrm{f}}\left|y-y_{0}\right|}$ and $v_{n}^{\mathrm{f}}=\sqrt{k_{\mathrm{p}_{\mathrm{f}}}^{2}-k_{z}^{2}-k_{n}^{2}}$ with $\operatorname{Im}\left(v_{n}^{\mathrm{f}}\right) \leq 0$

The solid displacement potentials are expressed as

$\phi=E_{\mathrm{a}} \sum_{n=-N}^{n=+N}\left(E_{\mathrm{b}} A_{n}^{\mathrm{f}}\right) E_{\mathrm{d}}$

$\psi_{x}=E_{\mathrm{a}} k_{z} \sum_{n=-N}^{n=+N}\left(\frac{-E_{c}}{\gamma_{n}} C_{n}^{\mathrm{f}}\right) E_{\mathrm{d}}$

$\psi_{y}=0$

$\psi_{z}=E_{\mathrm{a}} \sum_{n=-N}^{n=+N}\left(\frac{k_{n}}{\gamma_{n}} E_{c} B_{n}^{\mathrm{f}}\right) E_{\mathrm{d}}$

while the fluid pressure potential is given by the expression

$\phi_{\text {fluid }}=-\frac{\mathrm{i}}{L_{x}} \sum_{n=-N}^{n=+N}\left[\left(\frac{-\alpha_{\mathrm{f}}^{2}}{\omega^{2} \lambda_{\mathrm{f}}}\right) \frac{E_{\mathrm{f}}}{v_{n}^{\mathrm{f}}} D_{n}^{\mathrm{f}}\right] E_{\mathrm{d}}$,

when $y<0$

where $\omega$ is the frequency of the load, $k_{z}$ is the wavenumber in $z$ andi $=\sqrt{-1}$. Also in these expressions $E_{\mathrm{a}}=1 /\left(2 \rho \omega^{2} L_{x}\right)$, $E_{\mathrm{b}}=\mathrm{e}^{-\mathrm{i} v_{n} y}, E_{c}=\mathrm{e}^{-\mathrm{i} \gamma_{n} y}, E_{\mathrm{f}}=\mathrm{e}^{-\mathrm{i} v_{n}^{\mathrm{f}} y}$ and $k_{n}=\left(2 \pi / L_{x}\right) n$, $v_{n}=\sqrt{k_{\mathrm{p} 1}^{2}-k_{z}^{2}-k_{n}^{2}}$ with $\operatorname{Im}\left(v_{n}\right) \leq 0, \gamma_{n}=\sqrt{k_{\mathrm{s} 1}^{2}-k_{z}^{2}-k_{n}^{2}}$, with $\operatorname{Im}\left(\gamma_{n}\right) \leq 0, k_{\mathrm{p} 1}=\omega / \alpha_{1}, k_{\mathrm{s} 1}=\omega / \beta_{1}, \alpha_{1}=\sqrt{\left(\lambda_{1}+2 \mu_{1}\right) / \rho_{1}}$ and $\beta_{1}=\sqrt{\mu_{1} / \rho_{1}}$ are the velocities for P (pressure) waves and $\mathrm{S}$ (shear) waves, respectively, $\lambda_{1}$ and $\mu_{1}$ are the Lamé constants and $\rho_{1}$ is the mass density of the elastic medium. Meanwhile, $v_{n}^{\mathrm{f}}=\sqrt{k_{\mathrm{p}_{\mathrm{f}}}^{2}-k_{z}^{2}-k_{n}^{2}}$ with $\operatorname{Im}\left(v_{n}^{\mathrm{f}}\right) \leq 0, k_{\mathrm{p}_{\mathrm{f}}}=\omega / \alpha_{\mathrm{f}}, \alpha_{\mathrm{f}}=\sqrt{\lambda_{\mathrm{f}} / \rho_{\mathrm{f}}}$ is the acoustic (dilatational) wave velocity of the medium, $\lambda_{\mathrm{f}}$ is the fluid Lamé constant and $\rho_{\mathrm{f}}$ is the mass density of the fluid. $A_{n}, B_{n}, C_{n}$, and $D_{n}$ are as yet unknown coefficients to be determined from the appropriate boundary conditions, so that the field produced simultaneously by the source and surface terms should produce $\sigma_{y x}^{\mathrm{s}}=\sigma_{y x}^{\mathrm{f}}=0, \sigma_{y z}^{\mathrm{s}}=\sigma_{y z}^{\mathrm{f}}=0, \sigma_{y y}^{\mathrm{s}}=\sigma_{y y}^{\mathrm{f}}$ and $u_{y}^{\mathrm{s}}=u_{y}^{\mathrm{f}}$ at $y=0$. Imposing the four stated boundary conditions for each value of $n$ a system of four equations in the four unknown constants is found. This is a straightforward procedure, but the details are rather complex, and so are not presented here. The final system of equations is

$\left[a_{i j}^{\mathrm{f}} i=1,4 ; j=1,4\right]\left[c_{i}^{\mathrm{f}} i=1,4\right]=\left[b_{i}^{\mathrm{f}} i=1,4\right]$

with $\left[a_{i j}^{\mathrm{f}} i=1,4 ; j=1,4\right]$

$a_{11}^{\mathrm{f}}=-2 v_{n} \quad a_{12}^{\mathrm{f}}=\frac{-k_{n}^{2}}{\gamma_{n}}+\gamma_{n} \quad a_{13}^{\mathrm{f}}=\frac{-k_{z}^{2}}{\gamma_{n}} \quad a_{14}^{\mathrm{f}}=0$

$a_{21}^{\mathrm{f}}=-2 v_{n} \quad a_{22}^{\mathrm{f}}=\frac{-k_{n}^{2}}{\gamma_{n}} \quad a_{23}^{\mathrm{f}}=\frac{-k_{z}^{2}}{\gamma_{n}}+\gamma_{n} \quad a_{24}^{\mathrm{f}}=0$ $a_{31}^{\mathrm{f}}=\left(-k_{\mathrm{s} 1}^{2}-2 v_{z n}^{2}\right) \quad a_{32}^{\mathrm{f}}=-2 k_{n}^{2} \quad a_{33}^{\mathrm{f}}=-2 k_{z}^{2} \quad a_{34}^{\mathrm{f}}=\frac{\mathrm{i} 2 \rho_{1} \omega^{2}}{v_{n}^{\mathrm{f}} \mu_{1}}$

$a_{41}^{\mathrm{f}}=-\mathrm{i} v_{n} \quad a_{42}^{\mathrm{f}}=\frac{-\mathrm{i} k_{n}^{2}}{\gamma_{n}} \quad a_{43}^{\mathrm{f}}=\frac{-\mathrm{i} k_{z}^{2}}{\gamma_{n}} \quad a_{44}^{\mathrm{f}}=\frac{2 \rho_{1} \omega^{2}}{k_{\mathrm{p}_{\mathrm{f}}}^{2} \lambda_{\mathrm{f}}}$

$\left\lfloor c_{i}^{\mathrm{f}} i=1,4\right\rfloor$

$c_{1}^{\mathrm{f}}=A_{n}^{\mathrm{f}} \quad c_{2}^{\mathrm{f}}=B_{n}^{\mathrm{f}} \quad c_{3}^{\mathrm{f}}=C_{n}^{\mathrm{f}} \quad c_{4}^{\mathrm{f}}=D_{n}^{\mathrm{f}}$

$\left\lfloor b_{i}^{\mathrm{f}} i=1,4\right\rfloor$

$b_{1}^{\mathrm{f}}=0 \quad b_{2}^{\mathrm{f}}=0 \quad b_{3}^{\mathrm{f}}=\frac{-\mathrm{i} \rho_{1} \omega^{2}}{v_{n}^{\mathrm{f}} \mu_{1}} E_{\mathrm{f} 1} \quad b_{4}^{\mathrm{f}}=\frac{\rho_{1} \omega^{2}}{k_{\mathrm{p}_{\mathrm{f}}}^{2} \lambda_{\mathrm{f}}} E_{\mathrm{f} 1}$

with $v_{z n}=\sqrt{-k_{z}^{2}-k_{n}^{2}}$ and $E_{\mathrm{f} 1}=\mathrm{e}^{-\mathrm{i} v_{n}^{\mathrm{f}} y_{0}}$.

Once the constants have been found, the pressures associated with the surface terms may be calculated using the equations that relate potentials to pressures. The Green's functions for the fluid formation are then obtained from the sum of the source terms and these surface terms. Once this has been done, the final expression for the pressure field in the fluid medium is obtained in the following form

$$
\begin{aligned}
\sigma^{\mathrm{fs}}= & \frac{-\mathrm{i}}{4} H_{0}^{(2)}\left(k_{\alpha_{\mathrm{f}}} \sqrt{\left(x-x_{0}\right)^{2}+\left(y-y_{0}\right)^{2}}\right) \\
& -\frac{\mathrm{i}}{L_{x}} \sum_{n=-N}^{n=+N}\left(\frac{E_{\mathrm{f}}}{v_{n}^{\mathrm{f}}} D_{n}^{\mathrm{f}}\right) E_{\mathrm{d}}, \quad \text { when } y<0
\end{aligned}
$$

Notice that, if $k_{z}=0$ is used, the system of equations derived above is reduced to three unknowns, leading to the $2 \mathrm{D}$ response.

\section{References}

[1] Beranek LL, Vér IL. Noise and vibration control engineering. New York: Wiley/Interscience; 1992.

[2] Barry TM, Reagan JA. FHWA highway traffic noise prediction model. US Federal Highway Administration, Washington DC, USA. Report FHWA-RD-77-108; 1978.

[3] FHWA highway traffic noise model. US Federal Highway Administration, Washington DC, USA. Report FHWA-PD-96010; 1998.

[4] Hanson CE, Saurenman HJ, Anderson GS, Towers DA. Guidance manual for transit noise and vibration impact assessment. US Urban Mass Transportation Administration, Washington DC, USA. Report UMTA-DC-08-9091-90-1; 1990.

[5] Lam YW. Using Maekawa's chart to calculate finite length barrier insertion loss. Appl Acoust 1994;42:29-40.

[6] Muradali A, Fyfe KR. A study of 2D and 3D barrier insertion loss using improved diffraction-based methods. Appl Acoust 1998;53: 49-75.

[7] Filippi P, Dumery G. Etude théorique et numérique de la diffraction par un écran mince. Acustica 1969;21:343-59.

[8] Terai T. On calculation of sound fields around three-dimensional objects by integral equation methods.. J Sound Vib 1980;69:71-100.

[9] Kawai Y, Terai T. The application of integral equation methods to the calculation of sound attenuation by barriers. Appl Acoust 1990;31: $101-17$.

[10] Duhamel D. Efficient calculation of the three-dimensional sound pressure field around a noise barrier. J Sound Vib 1996;197(5): $547-71$. 
[11] Duhamel D, Sergent P. Sound propagation over noise barriers with absorbing ground. J Sound Vib 1998;218(5):799-823.

[12] Morgan PA, Hothersall DC, Chandler-Wilde SN. Influence of shape and absorbing surface-a numerical study of railway barriers. J Sound Vib 1998;217(3):405-17.

[13] Lacerda LA, Wrobel LC, Mansur WJ. A dual boundary element formulation for sound propagation around barriers over an infinite plane. J Sound Vib 1997;202:235-347.

[14] Lacerda LA, Wrobel LC, Power H, Mansur WJ. A novel boundary integral formulation for three-dimensional analysis of thin acoustic barriers over an impedance plane. J Acoust Soc Am 1998;104(2):671 -8.

[15] Jean P, Defrance J, Gabillet Y. The importance of source type on the assessment of noise barriers. J Sound Vib 1999;226(2):201-16.

[16] Godinho L, António J, Tadeu A. 3D sound scattering by rigid barriers in the vicinity of tall buildings. J Appl Acoust 2001;62(11):1229-48.

[17] Bouchon M, Aki K. Discrete wave-number representation of seismic source wavefields. Bull Seismol Am 1977;67:259-77.

[18] Tadeu A, António J. 2.5D Green's functions for elastodynamic problems in layered acoustic and elastic formations. J Comput Model Engng Sci 2001;2(4):477-95.

[19] Tadeu A, Kausel E. Green's functions for two-and-a-half dimensional elastodynamic problems. J Engng Mech, ASCE 2000;126(10):1093-7.

[20] Pedersen H, Sánchez-Sesma F, Campillo M. Three-dimensiona scattering by two-dimensional topographies. Bull Seismol Soc Am 1994;84:1169-83.

[21] Papageorgiou A, Pei D. A discrete wavenumber boundary element method for study of 3-D response of 2-D scatterers. Earthq Engng Struct Dyn 1998;27(6):619-38.
[22] Lamb H. On the propagation of tremors at the surface of an elastic solid. Philos Trans R Soc London 1904;A203:1-42.

[23] Bouchon M. Discrete wave number representation of elastic wave fields in three-space dimensions. J Geophys Res 1979;84:3609-14.

[24] Kim J, Papageorgiou A. Discrete wavenumber boundary element method for 3-D scattering problems. J Engng Mech ASCE 1993; 119(3):603-24.

[25] Beskos D. Boundary element methods in dynamic analysis, Part II (1986-1996). Appl Mech Rev 1997;50(3):149-97.

[26] Manolis G, Beskos D. Boundary element methods in elastodynamics. London: Unwin Hyman (sold to Chapman \& Hall); 1988.

[27] Tadeu A, Santos P, Kausel E. Closed-form integration of singular terms for constant, linear and quadratic boundary elements. Part I. SH wave propagation. EABE: Engng Anal Bound Elem 1999;23(8): $671-81$

[28] Tadeu A, Santos P, Kausel E. Closed-form integration of singular terms for constant, linear and quadratic boundary elements. Part II. SV-P wave propagation. EABE: Engng Anal Bound Elem 1999; 23(9):757-68.

[29] Phinney R. Theoretical calculation of the spectrum of first arrivals in layered elastic mediums. J Geophys Res 1965;70:5107-23.

[30] Bouchon M, Aki K. Time-domain transient elastodynamic analysis of 3D solids by BEM. Int J Numer Meth Engng 1977;26:1709-28.

[31] Tadeu A, António J. Acoustic insulation of single panel walls provided by analytical expressions versus the mass law. J Sound Vib 2002; 257(3):457-75.

[32] Farinha J, Reis A. Tabelas Técnicas. Lisboa: Edições técnicas ETL Lda; 2000. 